\title{
Supporting Information for: Enhanced Spontaneous Emission Rates for Single Isoelectronic Luminescence Centers in Photonic Crystal Cavities
}

Ruoxi Wang,,${ }^{* 1}$ Michio Ikezawa, ${ }^{1}$ Yoshiki Sakuma, ${ }^{2}$ Hiroyuki Takeda, ${ }^{2}$

Naoki Ikeda, ${ }^{2}$ Yoshimasa Sugimoto, ${ }^{2}$ Kazuaki Sakoda, ${ }^{2}$ Yuuta Yamada, ${ }^{2}$ and Yasuaki Masumoto ${ }^{1}$

${ }^{1}$ University of Tsukuba, 1-1-1 Tennodai, Tsukuba, Ibaraki 305-8571, Japan

${ }^{2}$ National Institute for Materials Science, 1-1 Namiki, Tsukuba, Ibaraki 305-0044, Japan

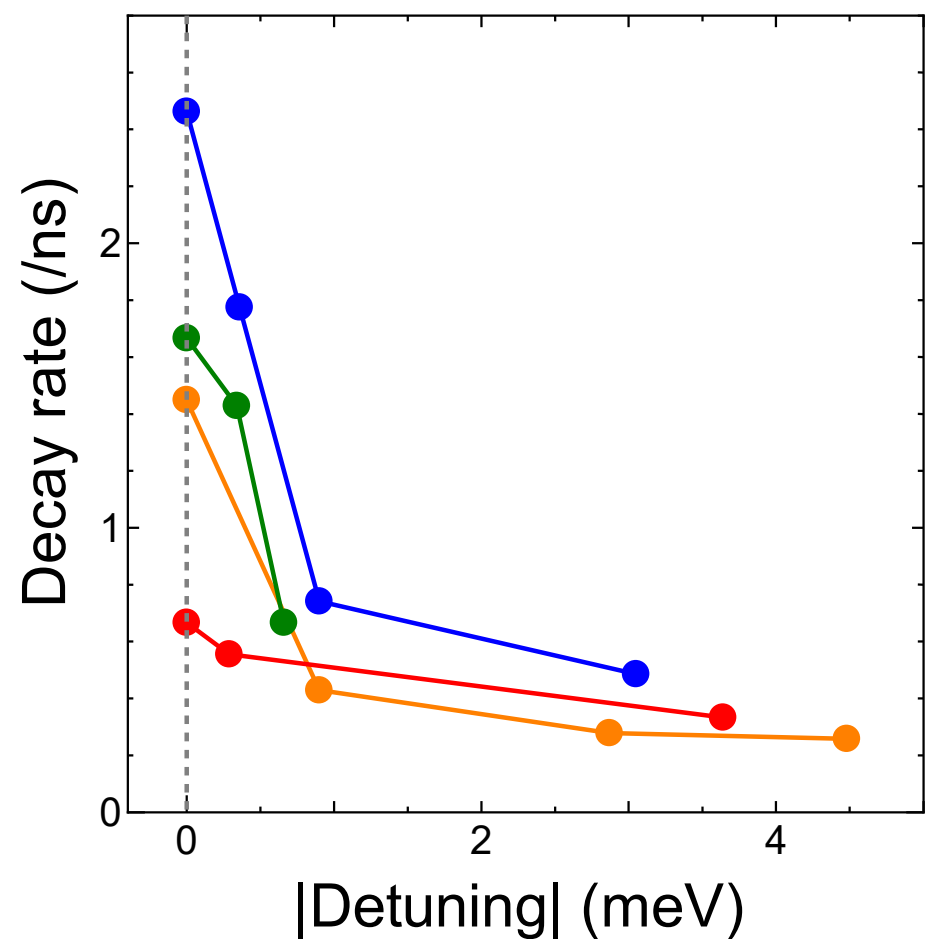

Figure S1. Dependence of the decay rates on the absolute value of the detuning for four PCCs. The blue data is the same as the data plotted in figure 3 (a). These decay rates tend to decrease monotonically with increasing absolute value of the detuning. 
(a)

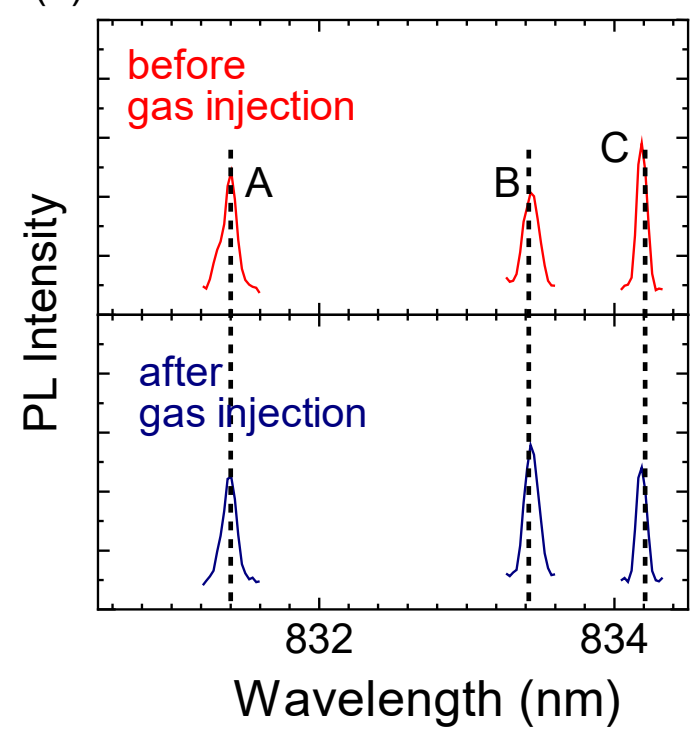

(b)

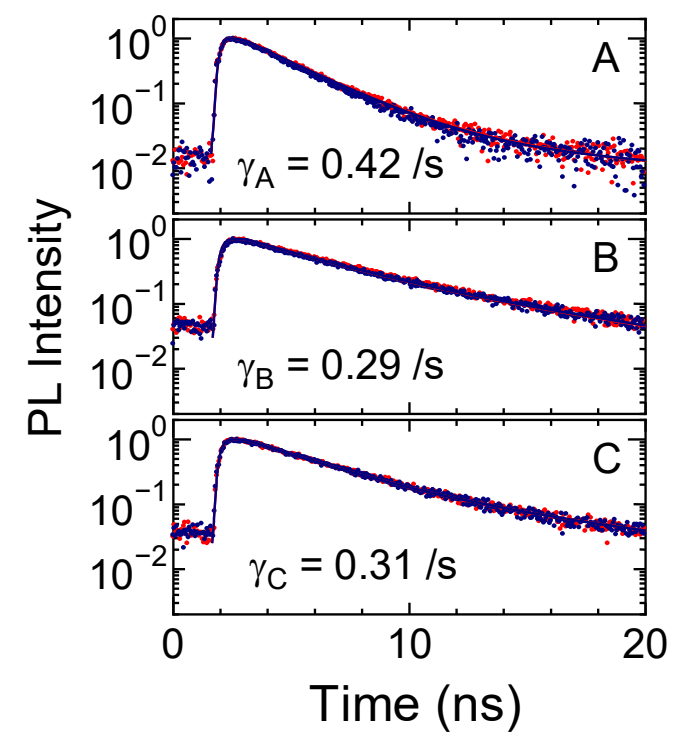

Figure S2. (a) PL spectra of three emitters $(A, B, C)$ outside PCCs before(red) and after(blue) gas injection at 5.3K. There is no significant change in these spectra. (b) The PL decay curves corresponding to these emitters. The decay rates of the emitters without cavity do not change at all before and after gas injection. 